\title{
Intervenção nutricional pautada na estratégia de oficinas em um serviço de promoção da saúde de Belo Horizonte, Minas Gerais
}

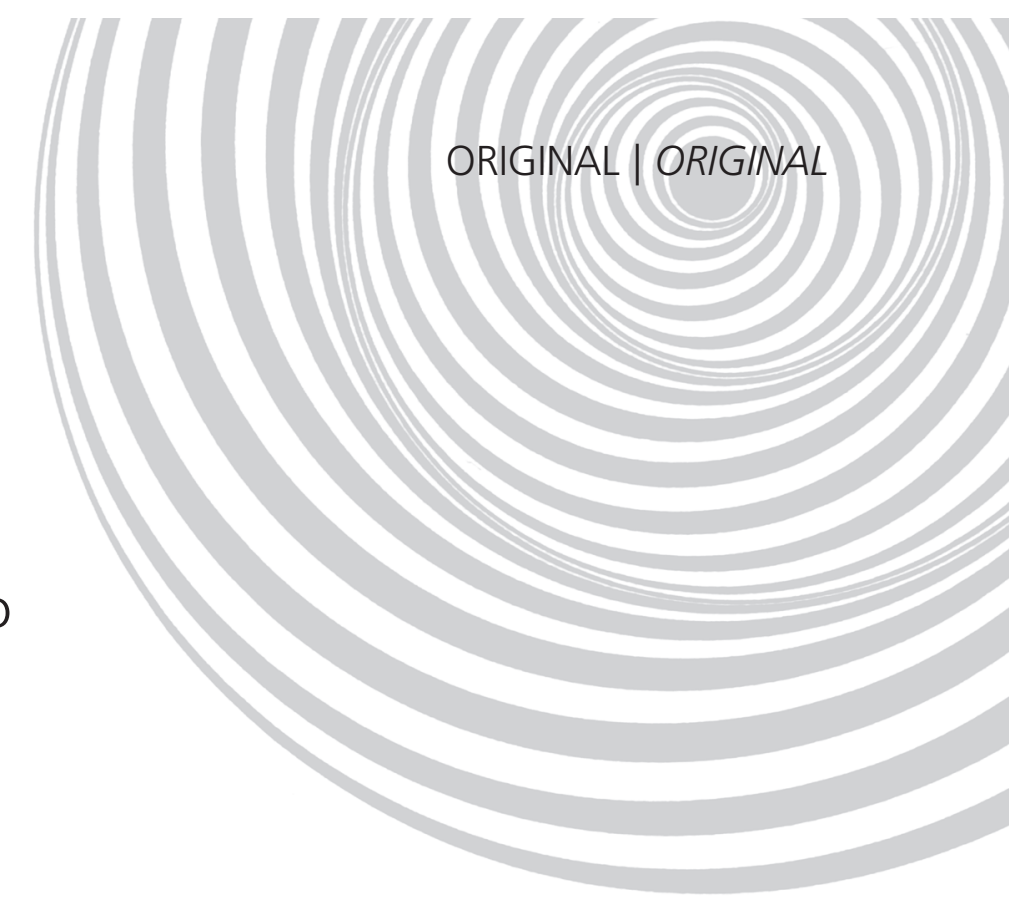

\author{
Nutritional intervention workshops in a \\ health-promotion service of Belo \\ Horizonte, Minas Gerais
}

Camila Pereira da SILVA'

Ariene Silva do CARMO'

Paula Martins HORTA ${ }^{1}$

Luana Caroline dos SANTOS ${ }^{1}$

RE S U M O

\section{Objetivo}

Avaliar as oficinas como uma estratégia de intervenção nutricional, com foco na aquisição de informações em nutrição e na percepção de mudanças de hábitos alimentares.

\section{Métodos}

Estudo de intervenção com usuários de uma Academia da Cidade de Belo Horizonte, Minas Gerais. Realizaram-se quatro oficinas, com frequência mensal e duração de trinta minutos cada uma, pautadas no "Guia Alimentar para a População Brasileira" e nos "Dez Passos para uma Alimentação Saudável". Aplicaram-se questionários para avaliar a aquisição de informações em nutrição antes e depois da execução das oficinas, investigar a percepção de mudanças de hábitos alimentares após três meses da intervenção e identificar as principais dificuldades verificadas nesse processo. Um observador apontou a viabilidade de execução das oficinas.

\section{Resultados}

O número de participantes das oficinas variou de 71 a 132. Houve maior ocorrência $(80 \%)$ de mulheres, com média de 55,8 $( \pm 10,6)$ anos. Identificou-se incremento estatisticamente significante na média total de acertos das questões do questionário de avaliação da aquisição de informações em três dos quatro encontros. Ademais,

\footnotetext{
1 Universidade Federal de Minas Gerais, Escola de Enfermagem, Departamento de Nutrição. Av. Prof. Alfredo Balena, 190, $3^{\circ}$ andar, Sala 324, Santa Efigênia, 30130-100, Belo Horizonte, MG, Brasil. Correspondência para/Correspondence to: PM HORTA. E-mail: <paulamhorta@gmail.com>.

Apoio: Fundação de Amparo à Pesquisa de Minas Gerais.
} 
90,9\% dos participantes referiram alguma mudança de hábito alimentar, destacando-se a redução no consumo de óleos, gorduras e frituras $(29,5 \%)$ e o aumento na ingestão de frutas e hortaliças $(22,5 \%)$. A redução no consumo de açúcares e doces foi apontada como a recomendação mais difícil de ser seguida (21,1\%). A avaliação do observador denotou a necessidade de modificar o espaço utilizado para as atividades.

\section{Conclusão}

A intervenção apresentou potencial para aquisição de informações em nutrição e mudança de hábitos alimentares, alterações que devem ser mais bem investigadas com a continuidade das ações.

Termos de indexação: Educação alimentar e nutricional. Educação em saúde. Hábitos alimentares. Promoção da saúde.

\section{A B S T R A C T}

\section{Objective}

To assess workshops as a nutritional intervention strategy, focusing on the transmission of nutrition information and perception of dietary changes.

\section{Methods}

The participants of this intervention study were users of a public gym (Academia da Cidade) in Belo Horizonte Minas Gerais. Four 30-minute workshops were held, one a month, based on the Brazilian Food Guide and Ten Steps to Healthy Eating. Questionnaires were administered to assess the participants' nutrition knowledge before and after the workshops, investigate perceived changes in dietary habits three months after the intervention, and identify the main difficulties associated with changing eating habits. One observer found the workshops viable.

\section{Results}

The number of participants in the workshops ranged from 71 to 132. The mean age of participants was $55.8 \pm 10.6$ years, and $80 \%$ were females. The number of correct answers in the questionnaires increased significantly in three of the four workshops. Moreover, $90.9 \%$ of the participants referred changing at least one eating habit, especially reducing the intake of oils, fats, and deep-fried foods (29.5\%), and increasing the intake of fruits and non-starchy vegetables (22.5\%). Reducing the intake of sugar and sweets was reported as the hardest recommendation to follow (21.1\%). The observer believes another area should be made available for the activities because, among others, the current area was too noisy.

\section{Conclusion}

The intervention has the potential of increasing participants' knowledge of nutrition and promoting dietary changes. These changes need to be better assessed as the workshops continue.

Indexing terms: Food and nutrition education. Health education. Food habits. Health promotion.

\section{N T R O D U Ç Ã O}

No Brasil, verifica-se elevada prevalência de hábitos alimentares inadequados, o que repercute negativamente na saúde dos indivíduos, pois favorece a ocorrência de Doenças Crônicas não Transmissíveis (DCNT), como obesidade, Diabetes Mellitus (DM) e Hipertensão Arterial Sistêmica $(\mathrm{HAS})^{1,2}$. Esse panorama sinaliza a necessidade de se desenvolverem estratégias de intervenção voltadas para a adoção de hábitos alimentares mais saudáveis ${ }^{2}$.
As ações educativas, na medida em que propiciam a participação ativa do sujeito na definição do seu estado de saúde e melhoram a relação profissional-usuário e os ambientes social e físico dos indivíduos, têm sido apontadas como um possível caminho para o enfrentamento das $\mathrm{DCNT}^{3}$. Dentre as modalidades de intervenções educativas em saúde, as oficinas se destacam pelo caráter participativo, inovador e singular ${ }^{4}$. Trata-se de um trabalho estruturado em grupos, com número variável de encontros, focalizado em torno de uma questão central em um contexto social. 
Os sujeitos são envolvidos de maneira integral, levando-se em conta as formas de pensar, sentir e agir 4 .

Em Belo Horizonte (MG), as oficinas têm sido utilizadas como uma estratégia de intervenção nutricional nas Academias da Cidade ${ }^{5}$. Similarmente a esses serviços, o Ministério da Saúde propõe implantar em nível nacional as Academias da Saúde ${ }^{6}$. Desse modo, conhecer a efetividade das ações realizadas nas Academias da Cidade torna-se bastante útil para a implantação desses novos serviços.

Nesse contexto, o presente estudo objetivou avaliar as oficinas como uma estratégia de intervenção nutricional, com foco na aquisição de informações em nutrição e na percepção de mudança de hábitos alimentares de usuários de uma Academia da Cidade de Belo Horizonte (MG). Além disso, o estudo propôs-se a avaliar a viabilidade de executar essa intervenção nesse serviço, considerando-se sua estrutura física e seus recursos disponíveis.

\section{M É T O D O S}

Trata-se de um estudo de intervenção aberta e não controlada, realizado no segundo semestre de 2011, com duração total de quatro meses, conduzido com usuários de uma Academia da Cidade, em Belo Horizonte (MG).

Academias da Cidade é uma das estratégias da Prefeitura Municipal de Belo Horizonte para promover a saúde e a qualidade de vida em contextos de vulnerabilidade social e hábitos de vida inadequados ${ }^{5}$. Esse serviço oferece gratuitamente a prática regular de exercícios físicos e a orientação nutricional individual e coletiva para indivíduos residentes em sua área de abrangência ${ }^{5}$.

Os exercícios físicos apresentam frequência de realização de três vezes por semana, tendo cada aula a duração total de uma hora ${ }^{5}$. Já o acompanhamento nutricional se dá por atendi- mentos individuais e por oficinas. A intervenção individual é desenvolvida com adultos com sobrepeso $^{7}$ e idosos eutróficos ${ }^{8}$; os demais são encaminhados ao Núcleo de Apoio à Saúde da Família (NASF) para acompanhamento com equipe multiprofissional ${ }^{5}$. As oficinas são oferecidas mensalmente à totalidade dos usuários, independente da condição nutricional ou de qualquer critério de exclusão ${ }^{5}$.

As intervenções realizadas nas Academias da Cidade são elaboradas tendo em vista o perfil do público atendido, caracterizado por elevada prevalência de excesso de peso e DCNT, tal como verificado por Costa et al. ${ }^{9} \mathrm{em}$ Academia da Cidade pioneira em Belo Horizonte (MG). Os autores constataram a prevalência de $41,6 \%$ de HAS, $70,6 \%$ de excesso de peso e $67,6 \%$ de risco para complicações metabólicas associadas à obesidade.

A amostra considerada para este estudo foi composta por todos os usuários ativos da Academia da Cidade em questão que compareceram às oficinas no serviço entre os meses de agosto $\mathrm{e}$ novembro de 2011. As oficinas eram previamente agendadas e informadas aos participantes em um mural de informação. Na semana anterior à sua execução, as datas das oficinas eram reforçadas verbalmente no momento das aulas de prática de atividade física.

Não foi realizado cálculo amostral sobre o interesse em avaliar a intervenção com a totalidade dos usuários ativos no serviço.

\section{Intervenção nutricional coletiva: oficinas}

O referencial teórico adotado para a intervenção nutricional coletiva apoiou-se em Afonso 4 . O tema trabalhado foi "alimentação saudável", com base no "Guia Alimentar para a População Brasileira" e em "Os Dez Passos para uma Alimentação Saudável"10. Destaca-se que cada oficina abrangeu de três a quatro passos, selecionados conforme a similaridade do tema (Quadro 1). 
Quadro 1. Conteúdo teórico e metodologia adotada para as oficinas com usuários da Academia da Cidade. Belo Horizonte (MG), 2012.

\begin{tabular}{|c|c|c|c|}
\hline Tema & Objetivos & Conteúdo Teórico & Metodologia \\
\hline Alimentação saudável & $\begin{array}{l}\text { Abordar os princípios gerais da } \\
\text { alimentação saudável contidos } \\
\text { no "Guia alimentar para a po- } \\
\text { pulação brasileira". }\end{array}$ & $\begin{array}{l}\text { Princípios gerais da alimentação } \\
\text { saudável. }\end{array}$ & $\begin{array}{l}\text { Reflexão em grupo sobre "O que é } \\
\text { uma alimentação saudável", segui- } \\
\text { da de explanação do tema, adotan- } \\
\text { do recursos visuais (cartaz com figu- } \\
\text { ras demonstrativas e placas colori- } \\
\text { das com o nome dos princípios). }\end{array}$ \\
\hline $\begin{array}{l}\text { Fracionamento das re- } \\
\text { feições, atividade física e } \\
\text { consumo de água }\end{array}$ & $\begin{array}{l}\text { Abordar os passos } 1,9 \text { e } 10 \text { dos } \\
\text { "Dez passos para uma alimen- } \\
\text { tação saudável". }\end{array}$ & $\begin{array}{l}\text { Fracionamento adequado das re- } \\
\text { feições e recomendação de consu- } \\
\text { mo de água e prática de atividade } \\
\text { física. }\end{array}$ & $\begin{array}{l}\text { Dinâmica de perguntas e respostas, } \\
\text { seguida de explanação do coorde- } \\
\text { nador e apresentação de gravuras } \\
\text { demonstrativas. Participação de } \\
\text { educador físico para orientação so- } \\
\text { bre práticas alternativas de ativida- } \\
\text { de física no domicílio. }\end{array}$ \\
\hline $\begin{array}{l}\text { Grupos alimentares e } \\
\text { recomendações de } \\
\text { consumo }\end{array}$ & $\begin{array}{l}\text { Abordar os passos 2, 3, } 4 \text { e } 5 \\
\text { dos "Dez passos para uma ali- } \\
\text { mentação saudável". }\end{array}$ & $\begin{array}{l}\text { Recomendação de consumo dos } \\
\text { grupos alimentares: "cereais, pães, } \\
\text { raízes e tubérculos"; "frutas"; "hor- } \\
\text { taliças"; "leguminosas"; "carnes e } \\
\text { ovos"; e "leite e derivados". }\end{array}$ & $\begin{array}{l}\text { Explanação da temática com o } \\
\text { auxílio de uma pirâmide alimentar } \\
\text { flanelada que ilustra os grupos ali- } \\
\text { mentares, suas porções e recomen- } \\
\text { dações de consumo, seguida de di- } \\
\text { nâmica de confecção de um cartaz } \\
\text { com essas informações. }\end{array}$ \\
\hline $\begin{array}{l}\text { Açúcares e doces, óleo e } \\
\text { gorduras e sal }\end{array}$ & $\begin{array}{l}\text { Abordar os passos } 6,7 \text { e } 8 \text { dos } \\
\text { "Dez passos para uma alimen- } \\
\text { tação saudável". }\end{array}$ & $\begin{array}{l}\text { Recomendação de consumo de, } \\
\text { açúcares e doces, óleos e gorduras } \\
\text { e sal. }\end{array}$ & $\begin{array}{l}\text { Demonstração da recomendação de } \\
\text { sal, açúcar e óleo vegetal e do teor } \\
\text { destes ingredientes nos principais } \\
\text { alimentos consumidos pela popu- } \\
\text { lação. }\end{array}$ \\
\hline
\end{tabular}

As oficinas ocorreram no período de agosto a novembro de 2011, aplicadas, mensalmente, em seis turmas, com no máximo 30 participantes, totalizando quatro encontros com cada uma. Somente os indivíduos que compareceram nos dias de realização das oficinas compuseram a amostra. Devido à alta rotatividade do serviço e ao fato de a intervenção ser do tipo aberta, os sujeitos que participaram de cada um dos quatro encontros não foram necessariamente os mesmos. Desse modo, os usuários podem ter participado de uma, duas, três ou quatro oficinas.

As oficinas ocorreram ao ar livre, tiveram duração aproximada de trinta minutos e foram previamente elaboradas por acadêmicos de nutrição da Universidade Federal de Minas Gerais (UFMG) que utilizaram um roteiro que contemplava: público-alvo, objetivos, duração total, materiais necessários, etapas da oficina, discurso dos coordenadores, avaliação e acompanhamento da oficina e cronograma.

As atividades foram conduzidas por acadêmicos do curso de Nutrição da UFMG e nutricionistas treinados. Ressalta-se que, no segundo encontro (Quadro 1), houve a participação de um educador físico para abordar o tema "Recomendação de atividade física". Cada encontro teve a participação de um coordenador responsável pela orientação do conteúdo temático, interação com os participantes, coordenação das dinâmicas e discussões, e por um observador externo, responsável por monitorar a condução do grupo e fazer a avaliação subjetiva da oficina.

As oficinas tiveram início com a apresentação da equipe de Nutrição. Em seguida, aplicou-se o questionário de avaliação das informações prévias acerca da temática a ser trabalhada. Posteriormente, procedeu-se à exposição interativa 
sobre o tema, seguida por atividades lúdicas. Ao final da oficina, era reaplicado o questionário para avaliação das informações adquiridas, acrescido de espaços para sugestões e comentários.

\section{Avaliação das oficinas}

As oficinas foram avaliadas sob o aspecto tanto da aquisição de informações em nutrição quanto da percepção dos próprios indivíduos acerca das mudanças de hábitos alimentares. Além disso, foi averiguada a viabilidade de execução das oficinas, considerando-se a estrutura física do serviço e os recursos disponíveis por meio de um roteiro para o observador.

Os questionários utilizados para a avaliação da aquisição de informações foram preenchidos por cada participante e aplicados antes e imediatamente depois das oficinas. Cada questionário compunha-se de duas a quatro questões fechadas, com apenas uma alternativa correta. As questões foram elaboradas especificamente para o estudo e em consonância com os temas trabalhados nos encontros. Os usuários mais idosos ou aqueles que apresentavam alguma limitação física ou psíquica não responderam aos questionários devido à dificuldade apresentada para essa avaliação. Destaca-se que o questionário aplicado após o encerramento da oficina apresentava espaço para sugestões, comentários e críticas relativas à oficina.

Ao longo da realização das oficinas, um observador externo ficou responsável por realizar a avaliação do andamento das atividades por meio de um roteiro que contemplava: adequação do tempo disponível à oficina; cumprimento do plano proposto; grau de interesse dos participantes; entendimento do conteúdo apresentado, com base nas expressões faciais e falas; e anotação das dúvidas e dos comentários mais comuns dos participantes.

Buscando identificar a contribuição das oficinas para a mudança dos hábitos alimentares, após três meses do encerramento das atividades, os usuários foram convidados a responder a um questionário de seguimento autopreenchido acerca de sua percepção quanto às mudanças ocorridas no período. Esse tempo foi considerado devido às evidências de que somente após três meses as mudanças de comportamento são efetivadas $^{11}$. O questionário abordava as seguintes questões: "Você considera que mudou algum hábito alimentar após a participação nas oficinas?"; "Quais orientações você considerou mais difíceis de seguir?"; Houve repasse de alguma das informações trabalhadas nas atividades para outras pessoas (amigos, vizinhos e familiares)?"; "Houve aplicabilidade das informações adquiridas (indique em nota de zero a dez, sendo zero igual a nada e dez igual a tudo) em sua alimentação?"; e "Você gostaria que as oficinas tivessem continuidade no serviço?".

\section{Análise dos dados}

Procedeu-se à análise descritiva dos dados numéricos e à aplicação do teste de normalidade de Kolmogorov-Smirnov. As variáveis com distribuição normal foram apresentadas em média (Desvio-Padrão - DP) e as demais em mediana (valor mínimo; valor máximo).

A análise dos dados do Questionário de Aquisição de Informações em Nutrição foi realizada com foco na média e na proporção de acertos individuais às questões antes e depois da intervenção, mediante o teste $t$ de Student pareado e o teste McNemar, respectivamente. Para a avaliação do questionário de seguimento, utilizaram-se o teste Qui-quadrado e o teste exato de Fisher. O nível de significância estatística foi estabelecido em $5 \%(p<0,05)$.

Realizou-se a análise qualitativa da avaliação do observador e dos participantes, que contemplou a descrição da avaliação das oficinas, realizada por ambos, incluindo principais comentários, sugestões, dúvidas, observações e críticas. Por fim, descreveram-se as principais modificações dietéticas alcançadas referidas pelos usuários, 
652 | CP SILVA et al.

assim como as dificuldades encontradas para promover as mudanças alimentares, conforme apontado no questionário de seguimento.

Foram utilizados os programas Epi Info 6.04 para construção do banco de dados, e o Statistical Package for the Social Sciences (SPSS) for Windows versão 19.0 para análises estatísticas.

O estudo foi aprovado pelos Comitês de Ética em Pesquisa da Universidade Federal de Minas Gerais (UFMG) (Protocolo 103/07, de 25 de abril de 2007) e da Prefeitura Municipal de Belo Horizonte (Protocolo 087/2007, de 14 de janeiro de 2008) e atendeu às recomendações éticas da Resolução 196 do Conselho Nacional de Saúde, de 10 de outubro de 1996.

\section{RES U LTA D O S}

O número de participantes nas oficinas oscilou de 71 a 132 usuários (Tabela 1). Observou-se maior prevalência de mulheres $(80 \%)$, com idade média de 55,8 (DP:10,6) anos.

Identificou-se incremento estatisticamente significante na média total de acertos das oficinas cujos temas foram "Fracionamento das refeições, atividade física e consumo de água" (de 1,70 DP:0,88 para 2,50 DP:0,70, $p<0,001)$ ) "Grupos alimentares e recomendações de consumo" (de 1,60 DP:0,99 para 2,60 DP:1,20, $p<0,001)$; e "Açúcares e doces, óleos e gorduras e sal" $(1,70$ DP:1,01 para 2,50 DP:0,81, $p<0,001)$. Para a oficina que abordou o tema "Aspectos gerais da alimentação saudável", verificou-se redução da média de acertos (de 1,60 DP:0,59 para 1,50 DP:0,74, $p=0,040$ ).

Quando avaliadas isoladamente, as questões desse questionário apresentaram aumento estatisticamente significante $(p<0,05)$ da aquisição de informações dos usuários no que concerne à maior parte das questões, com exceção de aspectos associados a estilo de vida que favorecem uma ótima saúde $(p=1,00)$, definição de alimentação saudável $(p=0,125)$ e recomendação de consumo de sal $(p=0,227)$ (Tabela 1).

Quanto à avaliação das oficinas, verificou-se grande satisfação dos usuários com a intervenção, tendo em vista os comentários realizados durante a oficina e no questionário de avaliação.

Tabela 1. Distribuição percentual de acertos obtidos pelos usuários da Academia da Cidade antes e depois das oficinas no questionário de avaliação da aquisição de informações em nutrição. Belo Horizonte (MG), 2012.

\begin{tabular}{|c|c|c|c|}
\hline \multirow{2}{*}{ Conhecimentos específicos (por questão) } & \multicolumn{2}{|c|}{ Acertos (\%) } & \multirow{2}{*}{ Valor $p$} \\
\hline & Pré-teste & Pós-teste & \\
\hline \multicolumn{4}{|l|}{ Alimentação saudável (n=123) } \\
\hline Aspectos do estilo de vida que favorece uma ótima saúde & 99,1 & 99,0 & 1,000 \\
\hline Definição de alimentação saudável & 87,1 & 94,5 & 0,125 \\
\hline \multicolumn{4}{|l|}{ Fracionamento das refeições, atividade física e consumo de água $(n=132)$} \\
\hline Fracionamento das refeições & 65,6 & 93,7 & $<0,001$ \\
\hline Frequência de realização de atividade física & 30,7 & 72,4 & $<0,001$ \\
\hline Recomendação diária de água & 81,5 & 96,5 & $<0,001$ \\
\hline \multicolumn{4}{|l|}{ Grupos alimentares e recomendações de consumo $(n=108)$} \\
\hline Recomendação diária de consumo de hortaliças & 34,6 & 73,7 & $<0,001$ \\
\hline Recomendação diária de consumo de frutas & 74,1 & 92,7 & $<0,001$ \\
\hline Recomendação diária de consumo de leite e derivados & 41,3 & 61,9 & 0,020 \\
\hline Recomendação diária de consumo de cereais, pães, raízes e tubérculos & 9,8 & 48,4 & $<0,001$ \\
\hline \multicolumn{4}{|l|}{ Açúcares e doces, óleo e gorduras e sal $(n=71)$} \\
\hline Recomendação de consumo de açúcar & 47,8 & 87,9 & $<0,001$ \\
\hline Recomendação de consumo de óleo vegetal & 49,2 & 78,5 & $<0,001$ \\
\hline Recomendação de consumo de sal & 83,6 & 90,5 & 0,227 \\
\hline
\end{tabular}


Além disso, os usuários sugeriram aumentar a frequência e o tempo de duração das atividades a fim de se aprofundarem mais as discussões (Quadro 2).

Considerando a avaliação do observador, dentre as principais dúvidas apresentadas pelos participantes ao longo das oficinas, destacam-se aquelas relacionadas a itens específicos da alimentação saudável, a exemplo do conteúdo de alimentos em uma porção dos grupos alimentares, bem como de escolhas saudáveis, do grupo de óleos e gorduras (Quadro 2).

Constatou-se, ainda de acordo com a avaliação do observador, a necessidade de mo- dificar o espaço para as atividades, pois o que foi disponibilizado favoreceu a dispersão do grupo. A distância dos usuários em relação ao coordenador da oficina, a não possibilidade de organização do grupo em um círculo (mesas e banquinhos fixos) e a ocorrência de ruídos externos dificultaram o andamento das oficinas. Além disso, verificou-se que o tempo para sua realização foi suficiente somente nos dois primeiros temas trabalhados.

Após três meses do período de intervenção, 71 pessoas responderam ao questionário de seguimento de avaliação da percepção de mudança de hábitos alimentares: 17,5\%, 20\%,

Quadro 2. Principais dúvidas e comentários dos usuários da Academia da Cidade participantes das oficinas segundo autoavaliação e avaliação do observador externo. Belo Horizonte (MG), 2012.

\begin{tabular}{|c|c|c|}
\hline Oficina & Principais comentários & Principais dúvidas \\
\hline Alimentação saudável (n=123) & $\begin{array}{l}\text { "Foi muito importante esta oficina de Nutrição. } \\
\text { Aprendi muito sobre os alimentos, como me ali- } \\
\text { mentar bem e como cuidar dos alimentos". } \\
\text { "Sugiro, se possível, aumentar o tempo, para apro- } \\
\text { fundar comentários sobre os itens debatidos". } \\
\text { "Eu achei muito interessante". }\end{array}$ & $\begin{array}{l}\text { "Como devo sanitizar os alimentos?". } \\
\text { "Como identificar os alimentos com } \\
\text { qualidade nos supermercados?". } \\
\text { "Como variar os alimentos?". }\end{array}$ \\
\hline $\begin{array}{l}\text { Fracionamento das refeições, atividade } \\
\text { física e consumo de água }(n=132)\end{array}$ & $\begin{array}{l}\text { "É muito bom aprender novos conceitos sobre } \\
\text { vida saudável". } \\
\text { "As oficinas são ótimas, pois tiram nossas dúvi- } \\
\text { das. Parabéns!" } \\
\text { "As oficinas da nutrição deveriam funcionar uma } \\
\text { vez por semana". }\end{array}$ & $\begin{array}{l}\text { "Qual a frequência recomendada para } \\
\text { a prática de atividade física?". } \\
\text { "Quantas refeições devemos fazer por } \\
\text { dia e o que seria contabilizado como } \\
\text { refeição?". } \\
\text { "Como posso realizar atividade física } \\
\text { fora de casa?". }\end{array}$ \\
\hline $\begin{array}{l}\text { Grupos alimentares e recomendações } \\
\text { de consumo }(n=108)\end{array}$ & $\begin{array}{l}\text { "A oficina oferece muito esclarecimento a todos. } \\
\text { Espero que continue assim". } \\
\text { "Estão sendo muito boas as orientações de como } \\
\text { devemos alimentar durante o dia". }\end{array}$ & $\begin{array}{l}\text { "O que é uma porção de alimentos?". } \\
\text { "Quantas porções do grupo de alimen- } \\
\text { tos fontes de carboidratos podemos con- } \\
\text { sumir em um dia?". }\end{array}$ \\
\hline $\begin{array}{l}\text { Açúcares e doces, óleo e gorduras e sal } \\
(n=71)\end{array}$ & $\begin{array}{l}\text { "Foi muito boa a palestra. Podemos aprender mais } \\
\text { sobre a nossa alimentação". } \\
\text { "Menos óleo, menos sódio, menos açúcares é } \\
\text { igual a mais saúde". } \\
\text { "Aprendi a alimentar adequadamente". } \\
\text { "Deveria ter oficina de nutrição todos os dias de } \\
\text { exercício". }\end{array}$ & $\begin{array}{l}\text { "Como mesurar uma porção dos ali- } \\
\text { mentos?". } \\
\text { "Quais alimentos têm açúcar?". } \\
\text { "Qual o melhor tipo de óleo e margari- } \\
\text { na para usar?". }\end{array}$ \\
\hline
\end{tabular}


Tabela 2. Modificações dietéticas alcançadas autorreferidas e recomendações nutricionais consideradas como mais difíceis de serem seguidas pelos usuários da Academia da Cidade. Belo Horizonte (MG), 2012.

\begin{tabular}{|c|c|c|}
\hline Modificações dietéticas alcançadas autorreferidas & $\mathrm{n}$ & $\%$ \\
\hline Diminuir o consumo de óleo, gordura e frituras & 21 & 29,5 \\
\hline Aumentar o consumo de frutas e hortaliças & 16 & 22,5 \\
\hline Diminuir o consumo de açúcares, doces e refrigerantes & 15 & 21,1 \\
\hline Diminuir o consumo de sal refinado & 10 & 14,0 \\
\hline Reduzir o consumo do grupo de cereais, pães, raízes e tubérculos & 8 & 11,2 \\
\hline Fracionar as refeições & 7 & 9,8 \\
\hline Substituir o leite integral pelo leite desnatado & 6 & 8,4 \\
\hline Aumentar a ingestão de água & 4 & 5,6 \\
\hline Quantidade e qualidade das refeições (geral) & 4 & 5,6 \\
\hline Aproveitamento integral dos alimentos & 2 & 2,8 \\
\hline Não realizaram modificações dietéticas & 11 & 9,1 \\
\hline \multicolumn{3}{|l|}{ Orientações nutricionais consideradas como mais dificeis de serem seguidas } \\
\hline Reduzir o consumo de açúcares e doces & 15 & 21,1 \\
\hline Reduzir o consumo de óleo e frituras & 7 & 9,8 \\
\hline Aumentar a ingestão de água & 6 & 8,4 \\
\hline Fracionar as refeições & 6 & 8,4 \\
\hline Quantidade adequada dos grupos alimentares (geral) & 6 & 8,4 \\
\hline Diminuir o consumo do grupo dos cereais, raízes e tubérculos & 3 & 4,2 \\
\hline Reduzir o consumo de sal & 1 & 1,4 \\
\hline Aumentar o consumo de frutas & 1 & 1,4 \\
\hline
\end{tabular}

22,5\% e $40 \%$ participaram de uma, duas, três e quatro oficinas respectivamente. A mediana de nota autorreferida para a aplicação dos conceitos adquiridos nas oficinas na alimentação foi de 9,0 $(0,0-10,0)$. Dos indivíduos que responderam ao questionário, 90,9\% e $89,4 \%$, respectivamente, referiram ter mudado de hábito alimentar e ter repassado as informações adquiridas nas atividades educativas a outras pessoas. Adicionalmente, $98,5 \%$ gostariam que as oficinas tivessem continuidade.

Não foi verificada associação entre o número de oficinas de que o usuário participou com mudança de hábitos alimentares e repasse de informações do conteúdo da oficina a algum conhecido $(p>0,05)$.

As principais modificações dietéticas referidas pelos participantes relacionaram-se à redução do consumo de óleos e gorduras (29,5\%) e ao aumento do consumo de frutas e hortaliças $(22,5 \%)$ (Tabela 2). A redução do consumo de açúcares e doces foi apontada como a recomendação nutricional mais difícil de ser adotada
$(21,1 \%)$, seguida da redução de óleos e frituras $(9,8 \%)$ (Tabela 2).

\section{I S CUSS Ã O}

Os resultados deste estudo evidenciaram o potencial das oficinas para a aquisição de informações sobre nutrição e mudanças de hábitos alimentares entre usuários de uma Academia da Cidade. Além disso, destacou a grande aceitação e satisfação dos usuários com a intervenção, apesar das dificuldades encontradas.

A aquisição de informações sobre nutrição corrobora os achados de Cervato et al. ${ }^{12}$ em estudo de intervenção que avaliou uma ação educativa com duração de seis meses com adultos e idosos ( $n=44)$ de São Paulo (SP). No teste relativo às noções conceituais sobre nutrição, os autores observaram aumento estatisticamente significante da média de acertos: de 5,01 para 6,2612.

Outra intervenção conduzida com indivíduos diabéticos residentes em Belo Horizonte (MG) verificou a aquisição de informações sobre 
gerenciamento do DM após seis meses de intervenção educativa em grupo $(n=54)$ e individual $(n=50)$. Houve aumento da média de acertos ao questionário (de 9,2 DP:2,8 para 10,6 DP:2,3; $p=0,017$ ), sem diferenças entre os grupos de intervenção ${ }^{13}$.

Neste trabalho, apesar de ter sido constatado incremento da média total de acertos para a maior parte das oficinas, para aquela relacionada aos aspectos gerais da alimentação houve redução. Procedeu-se, então, à análise isolada dos acertos das questões, não sendo observadas alterações estatisticamente significantes. Argumenta-se que o alto nível de informações prévio identificado nessas questões pode justificar os resultados observados. Para próximos estudos, sugere-se a consideração do nível de conhecimento basal dos sujeitos ao se avaliar a aquisição de informações com intervenções educativas. A investigação pode ser realizada, por exemplo, com grupos focais ou pela aplicação de um questionário prévio à intervenção. A utilização de variáveis mais controladas para medir conhecimento (psicometria) também poderia ser adotada, objetivando alcançar resultados mais robustos.

Considerando os achados deste estudo e aqueles verificados na literatura, observa-se que as ações educativas em saúde podem capacitar indivíduos e grupos na construção de novos conhecimentos, conduzindo a uma prática consciente de comportamentos preventivos ou de promoção da saúde ${ }^{13}$. As ações ampliam as possibilidades de controle das doenças, de reabilitação e de tomada de decisões que favoreçam uma vida saudável ${ }^{3}$.

As orientações nutricionais fornecidas durante as oficinas repercutiram em alteração autorreferida do comportamento alimentar. Similarmente, Guimarães et al. ${ }^{14}$ em estudo de intervenção com 80 adultos com excesso de peso, identificaram aumento significativo do percentual médio do uso de temperos naturais e do número de refeições ao dia nos dois grupos após três meses de estudo.

O trabalho desenvolvido por Mendonça \& Lopes $^{15}$, com adultos e idosos da Academia da
Cidade pioneira de Belo Horizonte (MG), após sete meses de prática regular de exercícios físicos e aconselhamento nutricional individual e/ou em grupo, constatou: redução do uso de banha animal $(p<0,01)$; aumento do percentual de indivíduos com classificação normal para circunferência da cintura e incremento do consumo diário de verduras/legumes e leite/derivados $(p<0,01)^{15}$.

Dentre as principais alterações de hábitos alimentares referidas pelos participantes deste estudo, destacam-se redução do consumo de óleos, gorduras e frituras, açúcares, doces e refrigerantes e aumento do consumo de frutas e hortaliças. Tais alterações são importantes e podem favorecer a melhora do perfil de saúde dos usuários acompanhados pela Academia da Cidade, pela possibilidade de diminuir o risco de desenvolvimento de doenças cardiovasculares e complicações metabólicas e de contribuir para o alcance de um peso saudável ${ }^{16}$, o que é relevante, tendo em vista o perfil de saúde dos indivíduos atendidos por esse serviço9?

Os participantes das oficinas apontaram algumas orientações nutricionais como sendo mais difíceis de serem seguidas. Guimarães et al. ${ }^{14}$ identificaram a realização de refeições fora de casa $(46,7 \%)$ e a dificuldade em aplicar os conhecimentos na prática, principalmente em eventos sociais (33,3\%), como as principais barreiras à adoção de uma alimentação saudável. Dificuldades em mudar hábitos alimentares já enraizados, falta de informação, de conscientização e de apoio familiar, influência negativa dos meios de comunicação e supervalorização de determinados alimentos em detrimento de outros são alguns fatores que desfavorecem a adoção de uma alimentação mais saudável ${ }^{17}$.

Os mesmos hábitos alimentares citados pelos participantes como sendo mais difíceis de serem seguidos foram também os mais referidos como modificações dietéticas alcançadas, o que sugere que esses foram os temas que mais sensibilizaram os participantes a tentarem as mudanças, apesar das dificuldades encontradas. 
Transformar conhecimento científico em mudanças efetivas alimentares é um grande desafio, haja vista o caráter multifatorial do comportamento alimentar. Assim, argumenta-se que o simples fornecimento de informações para aumentar o conhecimento não é suficiente para a mudança comportamental, sendo importante valorizar as dimensões subjetivas e sociais em um processo de troca interpessoal, em que os profissionais auxiliem os indivíduos a desenvolverem a capacidade de enfrentar os problemas, para obter melhores condições de saúde ${ }^{18,19}$. Essas evidências podem explicar o porquê de nem todos os indivíduos terem mudado seus hábitos alimentares.

O modelo transteórico tem-se destacado por postular que a mudança comportamental não acontece de maneira direta e abrupta, e sim ao longo de um processo dinâmico no qual as pessoas vivenciam diversos níveis de motivação para a mudança. Os estágios de mudança propostos por esse modelo - pré-contemplação, contemplação, decisão, ação e manutenção, representam esses estágios de motivação para a mudança. Acredita-se que pessoas em distintos estágios possuem diferentes percepções e motivações, além de encontrarem distintas barreiras à mudança de comportamento, tendo, assim, necessidades específicas de aconselhamento ${ }^{18,19}$. Diante disso, sugere-se que as intervenções em saúde considerem o estágio de mudança em que cada sujeito se encontrava antes da realização da ação.

Quanto à avaliação da intervenção realizada pelos participantes, os achados estão em consonância com os verificados por Cervato et al. ${ }^{12}$, em que os participantes da ação educativa sentiram-se gratificados pela oportunidade de ter acesso à informação e reconheceram a validade das noções em nutrição adquiridas para sua saúde e qualidade de vida. Em estudo transversal realizado por Siqueira et al..$^{20} \mathrm{com}$ adultos e idosos das regiões Sul e Nordeste do País, observou-se que os participantes reconheceram e indicaram a necessidade de manter uma alimentação saudá- vel como principal medida para a manutenção da saúde.

A comparação dos achados deste estudo com os dos estudos disponíveis na literatura foi dificultada pela pouca disponibilidade de trabalhos que utilizaram as oficinas como estratégia de intervenção. Além disso, as diferenças metodológicas adotadas em algumas intervenções educativas também limitaram as discussões dos presentes achados. Apesar disso, tem-se a investigação conduzida por Castro et al. ${ }^{21}$ no Rio de Janeiro (RJ), que avaliou oito oficinas com 166 indivíduos e apresentou resultados também satisfatórios. Essas oficinas contemplaram uma etapa de vivência culinária, seguida de debate entre os participantes, discussão dos conceitos surgidos do debate e avaliação de todo o projeto. A análise de discurso constatou que os textos distribuídos ao longo da intervenção foram lidos, as receitas e dicas de culinária foram experimentadas e esses materiais foram partilhados com os pares dos envolvidos. Além disso, foi recorrente o relato de que muitas experiências proporcionadas pelo projeto haviam sido incorporadas pelos participantes em sua prática alimentar pessoal e familiar $^{21}$. Outras investigações semelhantes às desenvolvidas pelo grupo de pesquisadores do Rio de Janeiro e às relatadas neste estudo poderão contribuir para a compreensão do impacto de intervenções nutricionais pautadas na estratégia de oficinas.

Por fim, discutem-se a perda amostral verificada para a última oficina e a baixa participação dos usuários no questionário de seguimento do estudo, o que indica a elevada rotatividade do serviço. Esses achados denotam a dificuldade de se avaliarem a efetividade e o impacto de ações de intervenções em serviços de saúde devido às limitações de tempo, espaço e adesão ${ }^{22}$. Nesse sentido, cabe destacar que as principais dificuldades enfrentadas no desenvolvimento das oficinas se relacionaram ao reduzido tempo disponível para a execução das atividades e à inadequação do espaço físico.

Dentre as limitações do estudo, aponta-se o fato de as oficinas terem sido desenvolvidas 
em apenas um serviço, não refletindo todos os usuários acompanhados pelas Academias da Cidade de Belo Horizonte. Entretanto, sabe-se da homogeneidade do perfil dos usuários nas diferentes Academias da Cidade, independente da região do estudo 9 . Além disso, o estudo não avaliou o conhecimento prévio dos usuários acerca da temática trabalhada nas intervenções, o que impossibilitou o controle dessa variável nas análises realizadas. Similarmente, não foram identificadas as práticas alimentares dos usuários no momento anterior à intervenção, o que permitiu somente a investigação da percepção dos usuários acerca da mudança de hábitos alimentares ao final das atividades.

A ausência de um grupo-controle também se configurou como uma limitação do estudo. Argumenta-se que as investigações conduzidas em serviços de saúde raramente são desenvolvidas em condições ideais de ensaios clínicos randomizados controlados, considerando razões práticas e éticas. Em adição, estudos realizados em contextos de situação de "vida real" apresentam validade externa e podem ser interessantes para a implementação de intervenções em nível populacional, sobretudo em se tratando de políticas públicas de saúde ${ }^{23}$. Por fim, a intervenção não considerou o estágio de mudança de comportamento dos indivíduos e não se deve atribuir as mudanças de hábitos percebidas pelos usuários somente às oficinas, já que, paralelamente, havia outras intervenções nutricionais sendo realizadas na Academia da Cidade.

\section{O N C L U S Ã O}

O estudo contribuiu para minimizar a escassez de estudos de intervenção nutricional que adotam as oficinas como referencial teórico e concluiu que a intervenção proposta apresentou potencial para a aquisição de informações em nutrição e para a adoção de hábitos alimentares saudáveis. Ressalta-se a importância de dar continuidade a essas ações e de expandi-las a outros serviços de promoção da saúde, incluindo as recentemente propostas Academias da Saúde, corrigidas as limitações metodológicas e aquelas associadas à estrutura e aos recursos da Academia da Cidade.

\section{COLABORADORES}

CP SILVA, AC SILVA e PM HORTA participaram da concepção do estudo, coleta de dados, análise e interpretação dos dados e redação do manuscrito; LC SANTOS participou da concepção do estudo e revisão da redação do manuscrito.

\section{REFERÊNCIAS}

1. Instituto Brasileiro de Geografia e Estatística. Análise do consumo alimentar pessoal no Brasil. Rio de Janeiro: IBGE; 2011.

2. Monteiro CA, Levy RB, Claro RM, Castro IR, Cannon $G$. Increasing consumption of ultra-processed foods and likely impact on human health: Evidence from Brazil. Public Health Nutr. 2011; 14(1):5-13. doi: 10.1017/S1368980010003241.

3. Ezzati M, Riboli E. Can noncommunicable diseases be prevented? Lessons from studies of populations and individuals. Science. 2012; 337(6101):1482-7. doi: 10.1126/science.1227001.

4. Afonso MLM. Oficinas em dinâmicas de grupo na área de saúde. São Paulo: Casa do Psicólogo; 2006.

5. Dias MAS, Lopes ACS, Bicalho K, Mourão M, Alves MN, Evangelista PA, et al. Promoção à saúde e articulação intersetorial. In: Magalhães Junior HM, organizador. Desafios e inovações da gestão do SUS em Belo Horizonte: a experiência de 2003 a 2008. Belo Horizonte: Mazza Edições; 2010.

6. Brasil. Ministério da Saúde. Portaria $n^{\circ} 719$, de 7 de abril de 2011. Institui o Programa Academia Saúde no âmbito do Sistema Único de Saúde. Brasília: Ministério da Saúde; 2011 [acesso 2013 jul 17]. Disponível em: <http://bvsms.saude.gov.br/ bvs/saudelegis/gm/2011/prt0719_07_04_2011. html>.

7. World Health Organization. Physical status: The use and interpretation of anthropometry. Technical Report Series n 854. Geneva: WHO; 1995.

8. The Nutrition Screening Initiative. Incorporating nutrition screening and interventions into medical practice. A monograph for phycicians. Washington (DC): The American Dietetic Association; 1994. 
9. Costa BVL, Mendonça RD, Santos LC, Peixoto SV, Alves M, Lopes ACS. Academia da Cidade: um serviço de promoção da saúde na rede assistencial do Sistema Único de Saúde. Ciênc Saúde Colet. 2013, 18(1):95-102. DOI: 10.1590/S1413-812320130 00100011.

10. Brasil. Ministério da Saúde. Guia alimentar para a população brasileira: promovendo a alimentação saudável. Brasília: Ministério da Saúde. 2008.

11. Grace C. A review of one-to-one dietetic obesity management in adults. J Hum Nutr Diet. 2011; 24(1):13-22. doi: 10.1111/j.1365-277X.2010.0 1137.x.

12. Cervato AM, Derntl AM, Latorre MRDO, Marucci MFN. Educação nutricional para adultos e idosos: uma experiência positiva em Universidade Aberta para a Terceira Idade. Rev Nutr. 2005; 18(1):41-52. doi: 10.1590/S1415-52732005000100004.

13. Torres HC, Hortale VA, Schall V. A experiência de jogos em grupos operativos na educação em saúde para diabéticos. Cad Saúde Pública. 2003; 19(4): 1039-47. doi: S0102-311X2003000400026.

14. Guimarães NG, Dutra ES, Ito MK, Carvalho KMB. Adesão a um programa de aconselhamento nutricional para adultos com excesso de peso e comorbidades. Rev Nutr. 2010; 23(3):323-33. doi: S1415-52 732010000300001.

15. Mendonça RD, Lopes ACS. The effects of health interventions on dietary habits and physical measurements. Rev Esc Enferm USP. 2012; 46(3): 573-79 doi: S0080-62342012000300007.

16. Te Morenga L, Mallard S, Mann J. Dietary sugars and body weight: Systematic review and metaanalyses of randomized controlled trials and cohort studies. BJM. 2012; 346 [cited 2013 Jul 20]. Available from: <http://wwwbmj.com>. doi: 10.11 36/bmj.e7492.
17. Kapur K, Kapur A, Ramachandran S, Mohan V, Aravind SR, Badgandi M, et al. Barriers to changing dietary behaviour. J Assoc Physiscians India. 2008; 56(1):27-32.

18. Coleman MT, Pasternak RH. Effective strategies for behavior change. Prim Care. 2012; 39(2):281-305. doi: 10.1016/j.pop.2012.03.004.

19. Norcross JC, Krebs PM, Prochaska JO. Stages of Change. J Clin Psychol. 2011; 67(2):143-54. doi: 10.1002/jclp.20758.

20. Siqueira FV, Nahasi MV, Facchini LA, Silveira DS, Piccini RX, Tomasi E, et al. Fatores considerados pela população como mais importantes para manutenção da saúde. Rev Saúde Pública. 2009; 43(6): 961-71. doi: S0034-89102009005000066.

21. Castro IRR, Souza TSN, Maldonado LA, Caniné ES, Rotenberg S, Gugelmin SA. A culinária na promoção da alimentação saudável: delineamento e experimentação de método educativo dirigido a adolescentes e a profissionais das redes de saúde e de educação. Rev Nutr. 2007; 20(6):571-88. doi: S1415-52732007000600001.

22. Victora CG, Santos IS. Los siete pecados capitales de la evaluación del impacto. Gac Sanit. 2008; 22(1):1-7. doi: 10.1016/S0213-9111(08)76068-0.

23. Ramanathan S, Allison KR, Faulkner G, Dwyer JJ. Challenges in assessing the implementation and effectiveness of physical activity and nutrition policy interventions as natural experiments. Health Promot Int. 2008; 23(3):290-7. doi: 10.1093/heapro/dan 022. 